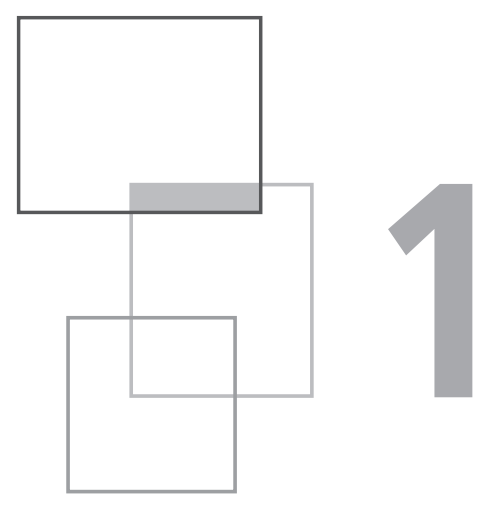

\title{
Informational
}

\section{propulsion of Society}

We are witnessing a huge push of the Digital in all areas of human activities. It reverses many practices and plunges all sectors of Society into deep questioning of their governance and even of their raison d'être. Thus, the macroeconomic sector is asking how to maintain full employment at a time when many reports announce the elimination of a large number of jobs due to the automation of tasks, which is induced by the massive deployment of the Digital. Should not the validity of the concept of "full employment" be revised in our times of the Digital?

The classical models of the challenges and effects of the Digital on Society are based on strategies fogged by fascinating visions, which have been raising expectations of immediate, almost infantile satisfaction of the usages that became possible thanks to the Digital. However, such frameworks have become obsolete to implement responsible strategies, due to the enormous push of the Digital.

Now it is the time to deploy both the Digital World together with explicit conscience of its societal environment and the Societal World together with a conscience responsible for its digital environment. This is the time of the Information World.

In the world of information, societal and digital responsibilities intermingle consciously and explicitly. It now becomes obvious that a common base is required that would allow comprehension between societal responsibilities and digital responsibilities. Moreover, it now seems to be inevitable that deeper explorations are necessary to deal with critical situations of questioning and analysing societal processes 
and to (re-)construct societal foundations. Indeed, these are not only the practices that the Information World seeks to reexamine in depth. It is more than anything the way of observing Society. The Information World encourages us to return to the foundations.

By taking into consideration the issues of a democratic Society, the approach suggested in this book shares the spirit of the Enlightenment. It offers to the parties involved in the refoundation processes to become real contributors to the extent of their talents, in a context where each one proves their critical thinking. They will require a common cognitive base to appreciate the contributions of others and to be pertinent, in order to co-construct the practices that should be consequently exercised through digital means. It is the purpose of this chapter to present such a base: the informational base.

\subsection{Classical approaches of the Digital World and the Societal World}

The Digital pushes significantly change existing practices by making them more efficient and more reliable, for example by automating administrative tasks. Moreover, they induce new practices, which extend the scope of social cohesion, in particular their legislative or regulatory aspects. Such situations become even more numerous under the unstoppable flood of digital pushes. This leads to profound transformations of enterprises, not only from the point of view of their organisation and their decision-making processes, but also by re-thinking their reasons for being, their principles of management, their rules and regulations, and even laws at the level of the whole Society. How will the Digital World and the Societal World face these new challenges?

\subsubsection{Classical approach of the Digital World}

The classical approach of the Digital World consists in continuously offering new digital pushes, in form of systems consisting of a mixture of technologies and software. It reveals a technical complexity that perhaps has no equivalent in any other engineering sector. However, beyond the purely technical aspects, this approach also asks a question regarding the future of the Digital in Society. It is thus essential to make a meeting in Society where a created system must be so useful for Society, that it will immediately assimilate this system. Moreover, such a meeting should allow and invite new generation of digital pushes, which would create new digital pushes, and so on.

If it is observed from inside the Digital, the development of the Information World might seem independent of Society. It is constructed brick by brick with high 
cognitive continuity enlightened with occasional breakthroughs. It is a continuous work of exploration, both meticulous and technical.

Yet, one should also take in consideration the social utility, promised at such meetings. Indeed, it is this utility that creates the legitimacy of the Digital for Society. Since this utility is only a potential, as it is not positioned as a prototype or a product, it is the task for digital demiurges to show evidence and even its inevitable effect for the progression of Society. This is how the Digital sells this utility for Society, in order to obtain budget for its development projects. Society, in its turn, buys it for its strategic reasons.

\subsubsection{Classical approach of the Societal World}

The classical approach of the Societal World only acknowledges digital pushes and their effects. It fumbles in its societal implementations. It tries to avoid its difficulties and responsibilities by sharing the visions of digital demiurges. The classical approach of the Societal World highlights the ethical aspects by denouncing digitalbased consequences of any and all kinds. Such consequences include for example those concerning the protection of the private sphere and effects on production line work by automation of cognitively coercive tasks. This classical approach denounces such serious consequences such as job losses because of the Digital; it denounces the loss of status, the general feeling of people that they are being superseded in the ability to meet their responsibilities and their concerns about the sustainability of employment.

This classical approach highly encourages one to challenge these issues. It attempts to legislate for this but is aware of the fragility of its proposals. Major digital projects are launched without their sustainability in value creation being ensured. This approach will always surrender to digital pushes and react to them while there is a general feeling that it always suffers from being unable to guide digital pushes.

\subsubsection{Facing profound transformations induced by digital pushes}

Even if it actually faces profound transformations induced by digital pushes in Society, the classical approach of the Digital does not feel concerned by them for at least three reasons:

- for each digital push, the digital approach also provides a system, the usefulness of which is perceived by Society as obvious, sometimes even inevitable. This approach is not concerned in implementation of this utility for Society. It does not want to get involved in proving this utility, but only observes it and improves its offer; 
- each ICT push is considered as independent from other ICT pushes. Thus, from its viewpoint, the Internet is just a network of computers which communicate with one another. Nevertheless, from the point of view of the Societal World, the Internet has a global perspective that connected computers are located worldwide. As far as the Digital is concerned, this fact is nothing else than just an opportunity to address and solve exciting problems, such as reliability, security and performance;

- digital works are of a technical nature and strive to find the most optimal solution. Since the most optimal solution for a problem represents a significant contribution to Society, the Digital considers that its social mission is accomplished.

Facing the same profound transformations, the classical approach of the Societal World does not have a sufficient background to integrate them as part of the politics of the progression of Society. It is helpless to evaluate what exactly is right and sustainable in transformations induced by digital pushes. The obvious visions of utility, which are presented by the Digital, can not serve it to develop a consistent position at the level of Society.

It feels constantly disempowered to deal with the continuous digital pushes.

This approach can only note which habits and practices have been installed in Society by using a digital system. If - at some point - it realises that some of its usages are contrary to the public interest, it must oppose them by using principles transcribed as laws, regulations or rules. In the Digital, however, it faces two forces: the force of accomplished facts of those habits and the force typical to the Digital, the digital pervasiveness, which was introduced by Lawrence Lessig (2000) in a magisterial way in the context of cyberspace.

And here is an example to illustrate this. Briefly, if Society, through its managers, wants to counter the effects of a digital system perceived as harmful, it must impose rules not only on citizens, but also on the Digital itself. It must require the Digital to integrate them in their digital systems. The stakes are high: if the rules are embedded in the systems, they can not be transgressed. However, in order to embed the rules into the system, the Digital must transform them into digital rules - which are not always possible - because they are the ones that will be programmed in the system, to ensure their validation. To do this, it will be necessary to change the code of the system, sometimes very significantly, - and this can even be unrealisable, unless it is completely redone by scratch. So no wonder the ICT decision-making persons are not very enthusiastic. They can take delaying positions, knowing that the strength of accomplished facts plays in their favour to bury these rules.

\subsubsection{Risks of these approaches}

These approaches, which are taken by the Digital World and the Societal World, are foreign to one another. They are walled in mutual indifference. Yet, it is crucial for the progression of Society to overcome this obstacle. 


\subsubsection{Dominant attitudes}

In both worlds, these approaches promote dominant, accepted as a reference, attitudes of the following types:

- to ignore the importance of the Digital in social development, by limiting the vision of the Digital to a simple tool, oriented to humans, of course;

- to consider the rising role of the Digital as a secondary factor of progression: it does not actually create wealth, it only automates processes;

- to consider the rising role of the Digital as a simple place of power where it seems enough to have fascinating digital visions and promises for Society, to be well able to speak about them, without being concerned with complex challenges to overcome, their impacts on Society, on its sustainability and employment politics, as well as on its real added value;

- to adopt the infantile attitude of consuming the Digital, of searching one's needs to be simply satisfied or even to expect oneself to be continuously amazed by the Digital.

These attitudes can be found in all sectors of Society. They contribute to lead the whole Society to potential failures of understanding, losing basic notions and meanings, lacking in sense, becoming profoundly destabilised by digital pushes, which are otherwise noted with fascination, having its members demobilised individually and collectively, by feeling outdated and afraid of being swept away by a cognitive tsunami.

\subsubsection{Cracks in the wall of indifference}

In its own development, the Digital confronts the need of a dialogue with the Societal World and does communicate with it in a very specific context. Some time ago, it was considered that its customers were nothing other than simple users of its systems. Thus, it was easy for the Digital to stand aside from occasional vulnerabilities of its systems that could even cause significant damages to customers: customers were obliged to sign a contract in which it was stipulated that the digital providers were exempt from any liability for errors caused by their systems!

Nowadays, however, the Digital puts its users in situations where other people, who initially have no commitment with it, are concerned by the use of its systems. These situations can be critical in the event of incidents or accidents: for example, when such situations are caused by a drone, an automatically driven car, a surgeon-robot, etc. These situations are so new, that they have not yet been the subject of laws, regulations or rules. Thus, because of its digital pushes, the Digital clashes with its own social non-legitimacy. Now it requires its own legal protection and - subsequently - its own political recognition that its ICT advances are of the public. It can only obtain the above by cooperating with the world of Society, which is the only legitimate instance to legislate it.

The Societal World must be prepared to assume this responsibility on behalf of the public interest. Before any negotiations, two important things should be considered. 
From one side, the Digital knows the future towards which it is leading. From another side, it is absolutely impossible to determine the entire multitude of social and societal situations induced by digital pushes. Therefore, the Societal World must retain the option to change the laws or regulations in response to changing situations that arise from usages and - if necessary - to impose them afterwards on the Digital.

\subsubsection{Enlightenment}

These approaches of digital pushes do not significantly consider a multitude of signals following opening of new businesses created thanks to the Digital, as well as new forms of interactions, exchanges, sharing, transactions, collaborations, co-designs, co-innovation between different individuals or legal entities in all sectors. Yet, there is an increasing number of people who are enthusiastically involved in them.

The same is true for many information systems in enterprises: the Digital seized them for budget reasons but it considered that it was only the application of the Digital. It wanted to impose its ways of doing, by transforming an information system into a solution! But it is a deadlock! However, there are certain information systems which are truly successful: their decision-making persons both from the digital side and the management side have successfully dissolved this wall of indifference.

For classical approaches of digital pushes, all these Enlightenment beacons are the result of the invisible hand and are therefore insignificant for the progression of Society. In fact, they are very fragile; their sustainability is not ensured whilst they construct the continuity of Society by endogenising the Digital. This invisible hand that has illuminated all these successes actually hides the great intelligence in the social digital engagement of talented people. Their success was without the voluntary knowledge of classical worlds: the Societal World and the Digital World.

\subsubsection{Conclusion}

These classical approaches of the Societal World and the Digital World have built a wall of indifference between them: they communicate only through visions, budgets and relations of power. Very often, they even appear as antagonists. This was a response to initial pushes of informatics in Society. Given the importance gained by the Digital in the progression of Society and in the face of even more digital discoveries, this response can no longer assume a consequent social development without deeply re-questioning it. This is the time to make visible this invisible hand!

\subsection{Informational approach}

These digital pushes have impacts on societal practices and, consequently, on the progression of Society. They arise in a dispersed manner, in different areas of Society. They appear in a disruptive manner, with soft impacts on Society. 
Given the risks and challenges, Society needs to transform these ICT pushes into real digital propulsions for the progression of Society. It is necessary that people with responsibility in the Societal World and the Digital World intermingle. It is indispensable to have an approach that recognizes this intermingling at its very heart, unlike previous classical approaches.

\subsubsection{Information World}

The specialists of the Societal World and the Digital World must collaborate to take on consequent responsibilities related to propulsion. However, these worlds have very different spaces of knowledge and methods of reasoning, as well as their challenges in the context of Society. It is nevertheless impossible to ask specialists from one of the two worlds to become specialists in the other, like for example ask managers to become IT specialists, or the other way around. By taking into account the challenges of Society, it is impossible to acrobatically juggle on the bridge over the gap that exists between these two worlds.

In fact, between these two worlds, there is the world of Information: it is a world of knowledge with its own knowledge and methods, unknown in the World of Society and the Digital World. It is focused on information. This concept of information is certainly shared by both worlds, but both typically only use it, without really paying attention to it. Moreover, the concept of information as such is not taught in most management and computer science programs. This concept has been highlighted as the conceptual model by researchers in engineering of information systems. Their research is published notably in the scientific conferences, such as INFORSID ${ }^{1}$ for French-speaking scientific audience and $\mathrm{CAiSE}^{2}$ at the international level.

The proposed informational approach makes the concepts of the Information world more visible, essential and inevitable. It considers that, in the context of the Information world, important decisions related to technological pushes regarding the propulsion of Society must be made. According to the informational approach, the Information World does not only serve as intermediary between the World of Society and the Digital World. The information takes the key role at the heart of development of multiple propulsions and provides their informational base. This informational base consists of a multitude of information models in the precise language of the Information World, all oriented towards the design and implementation of propulsions.

This informational approach proposes to build information services from its informational base. The builders of information services should be aware of the existence of the informational enlightenment introduced in the intention of this book and presented throughout the book. These builders must be inventive and critical.

1. http:// www.inforsid.fr.

2. https://www.caise19.it/. 
This informational base imports the knowledge, which is described in informational terms coming from all the domains concerned by the information service, in particular, from the Digital. This knowledge is essential for the designers of the informational base, so that their decisions regarding the informational base can be accurately passed on by the people responsible for the digital implementation and by the people responsible on the organisational level, who are in charge of activities that will be undertaken through information services. This knowledge is imported into the informational base according to the principles described below. The informational base is thus composed of four parts:

- the digital informational kernel. It includes all the knowledge of digital possibilities and difficulties, in particular those relating to the security of access to information, to the evolution of the informational base. This knowledge is the basis of informational models. For example, the conceptual models used in information systems come from the schema models used in technologies related to database management systems;

- the societal informational kernel. It contains essential knowledge to determine the activities and responsibilities induced by the information service, as well as the necessary skills and the principles of their organisation. It contains the model of activities and the related economical model, as well as the exhaustive list of activities and contributors;

- the informational regulatory kernel. It concerns generic knowledge, such as scientific and technical knowledge, standards, laws and regulations;

- the informational kernel. It concerns all informational models. It is in the informational kernel where all the knowledge is combined, in order to construct informational base.

The informational base is represented in the figure 1.1. by the yellow ellipse. The societal part is represented by the red ellipse, whilst the digital and the regulatory parts are depicted as blue and grey ellipses correspondingly. The other ellipses are related to the more general context of Societal, Digital, Regulatory and Society.

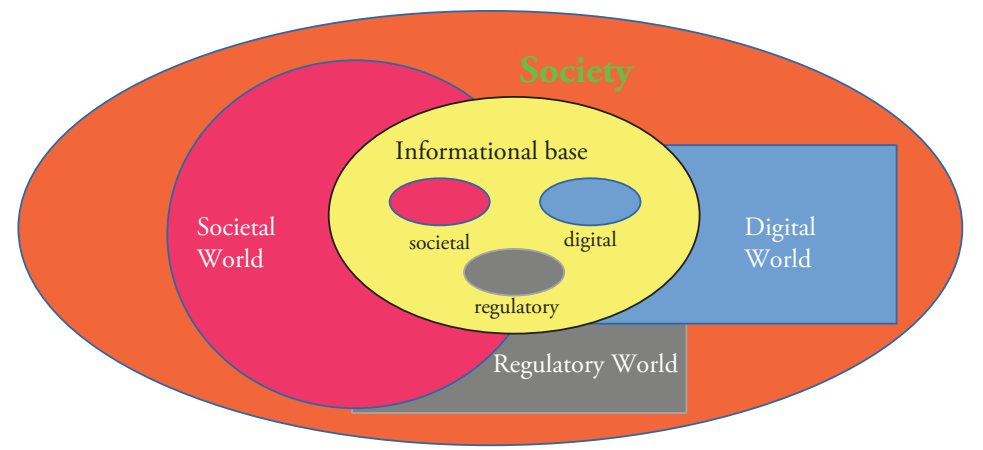

Figure 1.1. Informational base. 
The informational base is the steering place of the entire informational infrastructure, which has been developed to support an information service. This informational infrastructure includes of course the informational base, as well as all the digital and organisational implementations that have been put in place, in order to make operational this information service.

\subsubsection{Digital push of the Information World}

The Digital is always oriented towards the future. It only proposes rendezvous in the future to both enterprises and individuals by creating new digital technologies; the fact that both enterprises and individuals are present at these rendezvous by buying them, ensures the expansion of the Digital in all sectors of activities. It pushes the Societal World to also become oriented towards tomorrow, by putting the societal innovation in the centre of its missions and by being involved in innovation processes, in order to create, in its turn, new meetings in the future.

The consistent future of the Society with the Digital is not a hypothetical future. It is built very precisely, with an attention to informational detail, which does not really have an equivalent in the Societal World with such a wide extent. This special future is called tomorrow in this book. This is the informational construction that leads to a digital implementation: everything must be detailed, since, of course, no invisible hand can repair conceptual errors or approximations. It requires the underlying rigour of digital technologies. The Information World will push the Societal World to comply with this informational rigour: no mistake can be made, no vagueness is permitted. This is the world of the informational design which builds informational models.

The Information World uses the informational models entangled with each other to construct digital implementations. It pushes the Societal Information World to apprehend them, to participate in their construction, to make decisions in the choices of informational models, which all have their implication on the opportunities for actors working with the future system. It pushes the Societal World to enter into the world of informational models, where little by little meetings offered to tomorrow actors of the system are being formed. In order to overcome many difficulties, the methods of exploration of situations are required, which would allow to subdue their complexity by designing informational models.

In order to be efficient, the Information World requires that the phases of creative tension of design and implementation have to be protected, because they can last forever, given the amount of information that explorators must manage. In particular, the Information World pushes to avoid any undue delay in the decisions that are imperative for the development of the information service.

The Information World also pushes the Societal World to adopt a management approach, which gives a central place to the informational innovation. It also should take into account that the success of an information service requires many people to be involved into the process of design and implementation. The situations to overcome 
are so diverse and numerous. All the details have an important, sometimes even crucial, role. It is thus impossible that only one person controls or manages the entire process.

The Information World can not be embedded in the objectives, to which they should provide solutions. These meetings, which it sets tomorrow for individuals or enterprises, are expressed in terms of openness to discover the need in terms of creation and consolidation of activities. The Digital Information World pushes the Societal Information World to explore digital pushes and not to consume them. In addition, it pushes the Societal Information World to consider every informational implementation as still in the process of development and not as an end in itself or a final solution.

\subsubsection{Societal contra-push in the Information World}

In the context of the Information World, the digital push in the Societal World generates a societal contra-push in the Digital World. It might take multiple forms. Facing the digital pushes, the Societal Information World has the responsibility visà-vis Society, to manage and direct the evolution of information services or services that are supported in particular by the systems of these pushes. So, it pushes the Digital to provide it with the knowledge necessary to assume these responsibilities.

It also pushes it to provide interoperable systems, since the information, even if it is taken into account by different digital systems, nevertheless belongs to the same information service.

The Societal Information World pushes the Digital to provide evolutive systems that would enable it to carry out the necessary evolutions of the information service in response to continual explorations and continual adjustments to the evolution of the environment.

While, a digital push is primarily concerned with its system and its usefulness, particularly the satisfaction of generic users, the Societal Information World considers people who exercise their activities in the form of responsibilities or tasks. These are the actors. The responsibility of the Societal Information World lies in ensuring that they continue to exercise their activities within the information service efficiently. The situations intrinsic to their activities must be found in the information service available to them. It pushes the Information World to reason in terms of situations and to explore, design, achieve, evolve the interfaces between the actors and the information services.

Faced with a given situation, these actors need to have confidence in the information obtained from the system by having access to its source and evaluating its relevance. They need to have confidence that the information service faithfully reflects their actions. The Societal Information World pushes the Digital to be able to enrich all information with its provenance and its relevance, and to enrich each and every operation with the circumstances of its processing and with its effects.

While the Digital normally considers users to be isolated from each other in their intentions, the Societal Information World, on the contrary, takes into account the 
actors as participating in a collective activity to which everyone contributes. They share activities and information with people in the same enterprise or other enterprises. They need to have confidence in their knowledge of how exactly the other actors execute their activities through this information service. Thus, access operations offered by the Digital are not sufficient for the Societal Information World. It pushes the Digital to introduce the concept of the informational space for each actor: a list of different types of information and information treatments to which an actor has an access. Since actors are not isolated from each other, the informational space of an actor has a non-empty intersection with the informational spaces of several other actors. These informational overlaps should be viewed as objects of protocols governing the activities of each actor, in order to ensure consistency of the collective activity in which all these actors participate. In this way, the Societal Information World pushes the Digital to enable the implementation of these protocols of informational overlaps.

The Digital provides generic means of security at the level of each of its systems. The Societal Information World pushes the Digital to consider the level of informational security based on the relevant informational spaces. As the informational space of one actor can be supported by several systems, it also pushes the Digital to consider that informational security should be defined by multiple systems.

The Digital makes available generic consistency rules which it inherently validates. The Societal Information World has the responsibility of the regulatory kernel of the information service. It is composed of management rules of an enterprise, rules regarding the overlap protocols, norms, societal rules, various regulations and legislations. It has the responsibility to ensure compliance of information services with all these rules. Not all of them can be implemented in an information service. Therefore, their validation becomes the responsibility of actors. For other rules, the Societal Information World should develop their implementation within the system, as well as it should ensure a possibility for eventual modifications. It pushes the Digital to develop a real regulatory space allowing, on the one hand, to implement all these integrity rules and, on the other hand, to facilitate the evolution of their implementation in response of the evolution of the regulatory rules, in order to maintain compliance of these information services.

Finally, the Societal Information World pushes the establishment of methods of development and management of information services. These methods take into account not only digital pushes, but also the regulatory space, situations, evolutions and explorations, and deviate from linear methods, which start from goals to get solutions, which are practiced in the context of the indifference between the Societal World and the Digital World (\$ 1.1.4.1).

\subsubsection{Societal push in the informational context}

Society continually faces common situations and emerging situations. The answers to common situations are objects of standards and references that allow 
implementation and monitoring of societal operational practices, and would use these standards to respond to common situations. In contrast, emerging situations come from the aspirations of their members, unexpected events of all kinds, complexities, which have never been met before, information and relevant knowledge, which have not been accessible previously. There is no reference of societal practices to deal with them. Persons with responsibility often find themselves in the situations when their decision making would have consequent impacts on the future of Society, such as launching a legislative process.

All these situations lead to implement societal processes. The Digital has proven, however, that it can increase the efficiency of its own implementation. In this way, the classical approach advocates making decisions in the Societal World, without worrying about the Digital, and then turn to the Digital, in order to seek solution there. If one manages to destroy the wall of indifference between these two worlds, one would give an opportunity for the digital contra-push to take place, especially in emerging situations. Thus, instead of placing persons with responsibilities into risky situations to take important decisions, it puts them in position to lead a process of exploration, focusing on each step to implement societal processes, which are enriched thanks to the Digital. For this approach, it is necessary to establish the following chain of responsibilities:

$$
\text { Society } \Leftrightarrow \text { Societal } \Leftrightarrow \text { Information } \Leftrightarrow \text { Digital. }
$$

An example of such an approach is restructuring of an enterprise. Instead of planning restructuring an enterprise without taking into consideration all information services, which are already implemented in this enterprise, an exploratory approach (Opprecht and Léonard, 2014; Ralyté et al., 2016) considers their conceptual models and suggests how the conceptual models should evolve according to each step of the exploration of restructuring. In the exploratory approach, persons with responsibility are much closer to immediate impacts of restructuring and have more opportunities to refine their restructuring decisions in real time.

Another example is provided in the book of Fred Turner (2006), which presents the entanglement of aspirations in the Societal World and the Digital World. This entanglement is based on "the first key of the digital utopia", where the information has a central role for everything. When these two worlds intermingle, pushes and contra-pushes come in fusion to propel Society.

\subsubsection{Informational propulsion of Society}

Fred Turner describes the connivance of social and digital aspirations. They are intertwined to form meetings between actors of Society, and digital creators. The social aspirations of the actors of Society come in line with the digital aspirations of digital creators. The actors of Society seem to give sense to digital creativity; digital 
creators seem to make actors' aspirations concrete and realisable. Their intermingling starts playing the role of propulsion for the development of the Californian society.

The Information World has different amplitude. It does not only have its interest in digital enterprises. It focuses on Society in all its complexity and all its components. The Information World also suggests to intermingle the Societal World and the Digital World, but its purpose is to form informational propulsion in the very heart of Society.

\subsubsection{The force of the Information World}

The force of the Information World is to provide a framework helping to entangle digital pushes and social pushes. It is based on information and design. Information plays a central role. In the Digital, it describes anything that can be put in a binary form. In the Societal World, it holds an obvious essential place among all actors. It is in the centre of the Societal, in particular when it transcribes social aspiration at the societal level. The Information World gives it many forms: data, knowledge, information, rule, procedure, communication, as well as processes that allow establishing precise digital and societal designs.

The Information World channels the force typical to the Digital, which was previously presented $(\$ 1.1 .3)$, by mastering informational models, which make it possible to take the measure of this force on the Societal World. Once embedded in one or more digital systems, design models define the $\operatorname{code}^{3}$ which would induce dominant effects on the Societal World. Since this code has significant effects on Society, it is desirable to place its conception in a democratic context. The Information World allows it: by allowing the co-design and co-responsibility of the models at the informational level, it allows to establish coherent debates between actors of the Digital Information World and the Societal Information World, based on the accuracy of the informational models regarding the Societal World.

Thus, the Information World contributes to the legitimacy of information services supporting societal activities. This legitimacy has two facets:

- the first one, "true", concerns digital implementations of informational models from the point of view of its engineering dimensions, such as reliability, performance and security;

- the second one, "right", concerns societal implementations based on informational models in their organisational, managerial and efficiency dimensions to enable the actors to face the situations, which they encounter within the framework of their activities, within the framework of the corresponding laws, rules, norms, ethical codes, etc.

3. In reference to the article "Code is Law" previously cited (Lessig, 2000). 


\subsubsection{A vast space of innovations}

The Information World provides a vast space for informational innovations, since it concerns all sectors of Society. These innovations relate to all levels of Society, the strategic and tactical levels, the operational level and the level of regulations. Such innovations can not be only to descendant or ascendant, they can not be only reserved for experts to innovate, and they can not be reduced to a single domain, unless they hinder the progression of Society. Coming from everywhere, innovations are multi-sectoral, multi-disciplinary, and sometimes multi-national. All of them have an important obligation: to create value, manage information services, which are both true and right, and to support the societal changes they induce.

Everyone who is involved in the co-creation of information services is also involved in co-creation of informational models. However, by doing so, people are required to search for the sense of the information service. They may even find several senses. They detail and concretise the sense during the progress of works of co-creation, and then share it with more people, even outside of the co-creation team. Each of co-creators has the sense of contributing to the progression of Society.

\subsubsection{Risks of exclusion}

The other side of this vast space for informational innovations is the risk of informational exclusion: i.e. the inability of a person to capture one of these senses and connect to it. Such a person finds herself completely disoriented with this information service and has no cognitive references to ensure her responsibilities. This can lead a person to a panic situation. This informational exclusion is even deeper than digital exclusion, which concerns the impossibility to access the Digital or the impossibility to comprehend the principles of its usages.

There is even a more insidious and extensive form than informational exclusion on the level of Society. It is the informational exclusion of potential creators who are being robbed of informational innovation by others (probably more influential persons) that have the power to impose their solutions.

The persons with responsibility who do not understand the importance of the Information World in the progression of Society do not have an argument against it. They simply are not aware that if one reduces informational innovation to simple digital innovation, one can not take into consideration the societal innovation, which has an outstanding importance for the progression of their Society and is characterised by an important added value, including the economic one. They are not aware that the "solution" constructed in this way is built in a societal context of a different Society, thus such a "solution" just implicitly introduces this societal context in their Society.

The fight against informational exclusion is very difficult. It is the informational approach that enables to highlight it and helps to overcome it by creating informational commons. 


\subsubsection{Information common goods}

This situation closely resembles the so called "tragedy of the commons" (Hardin, 1968) in the context of the management of natural common goods, such as fisheries, forests, water sources. In this domain, the valuable research by Elinor Ostrom (1990) earned her the Nobel Prize in economics in 2009. It is important to build, nurture and continuously enrich the information common goods. Given the multitude of informational resources to inventory, their management can only be done through the contributory management, where each contributor should feel recognised both economically and socially. These information common goods create the base of all the digital economy.

\subsubsection{From cognitive disruption to cognitive continuity}

Under the leadership of the Information World, Society is expanding. It introduces a dimension of innovation that is related to a wide range of actors, not just researchers and $R \& D$ departments of enterprises as in the case of product innovation.

This Information World takes into account in an explicit way both common situations and emerging situations. The first ones, even if they are faced with unforeseen circumstances, nevertheless remain in a known conceptual framework, certainly being a subject to continuous improvements. The conceptual framework of the second ones is either incomplete, or unknown, or both. In this case, a process of exploration is initially required.

Obviously, the Information World takes into account the common situations, however, it is best known for taking into consideration these emerging situations. For people who are engaged in co-creation of a new information service, such situations seem to be disruptive. Not only do they seem to be disruptive - they are actually disruptive. However, in the societal framework and consequently in the social framework, it is essential that these situations reach sufficient maturity to be presented as a continuity, particularly to avoid informational exclusions. This is the responsibility of the Societal Information World to ensure this continuity, definitely not just through simple communication such as marketing on presenting a new product, but based on the sense, which served as a keystone for co-creators.

The Information World must thus manage concomitance of activities, facing both common and emerging situations. And whilst the management and economics of common situations give the impression of being well known, the management and economics of emerging situations still remain to be explored, especially to discover new activities allowing value creation and their implementation in Society.

\subsubsection{Properties of the informational approach: a vast space of explorations}

In conclusion, the Information World allows maintaining a coherent conversation between the Societal World and the Digital World. It does not have a simple role of 
intermediation between these two worlds. It is the Information World that governs the Digital of Society through its component of the Digital Information World. It is the Information World that governs the Societal World through its component of the Societal Information World. It is the Information World that has the responsibility of the sense of the progression of Society induced by its informational propulsions. It is also the responsibility of the Information World to ensure the cognitive cohesion between all its actors.

It offers a vast space of responsible informational innovations with true and right information services, which explicitly bring their value to Society. This vast area of innovations requires a significant number of contributors from diverse professional and social backgrounds who would participate in all activities of exploration and informational co-creation.

It contributes to provide legitimacy of the democratic nature to informational implementations, to fight against informational exclusion of any kind, by taking into account both the common situations and emerging situations, to clarify responsibilities between the areas of Society, the Societal World, the Digital World and the Information World.

Therefore, thanks to digital pushes and social pushes, as well as the corresponding contra-pushes induced by them, the Information World becomes a real booster of the progression of Society.

\subsection{Conclusion: towards information intelligence}

Classical approaches of the Societal World and the Digital World turn out outdated and unable to meet the enormous potential of the Digital to ensure the social development. These approaches even offer strategic alignment of these two worlds, meaning that the Digital World should probably align with the Societal World. This alignment - i.e. this disposition of different things placed one next to another one in a straight line - can only hinder the progression of Society by inhibiting the informational development. Moreover, it reduces the role of the members of Society as simple consumers of the Digital (sometimes even by infantilising them) or just digital developers. They are all excluded from the informational innovation. However, in its turn, Society itself deviates from the societal innovation and must consume the innovation developed by others. Such approaches can only identify the situations of digital exclusions, without even being able to detect informational exclusions. They can not claim they can achieve any of the properties of the informational approach, which were previously described.

In particular, facing digital pushes that continue to open new potentialities of activities in Society, the informational approach ensures Society avoids falling into the trap to "regulate everything $v$ s. let everything go on its on". Indeed, it offers no obstacle to digital creativity. In contrast, the informational approach focuses on the 
digital endogenisation in societal activities by taking into consideration digital ICT pushes, as well as societal contra-pushes.

It is a real information intelligence, which should be developed to provide cognitive references to all the people - and there are many such people - who are concerned by the Information World on its various levels. It can not follow solely pragmatic approaches, because the Information World gives pushes not only on the level of actions, events, organisations, or practices. It is not even only for management and economics. The foundations of Society, which were built without the Information World, should be reconsidered. For example, what is a University in an informationally expanded Society? What is a hospital in such a Society? It is for the information intelligence to suggest exploratory approaches by respecting democratic principles. In this way, the motto of the French Revolution "Liberty, Equality, Fraternity" takes a critical and concrete dimension to (re-)build societal foundations. This motto will serve again to establish a common basis of collective understanding of all refoundings to explore and implement. Thus, an extension of universal Human Rights in the informationally expanded world becomes particularly relevant and essential.

This information intelligence is based on scientific knowledge, some of it coming from hard sciences and some from humanities. This information intelligence takes into account multi-sectoral, multi-institutional, multi-disciplinary situations, in order to form perennial information common goods from which various information services can be constructed. It is the management of reticular responsibilities, the economy of contribution, the management of contribution that must be put in place to meet the challenges of informational potentialities available to Society, and especially the challenges of many initiatives of contributors, who are eager to participate in the progression of Society.

Moreover, information intelligence is interested in the distribution of responsibilities between the powers of Society - such as executive, legislative, judicial powers for a democratic state - extended now to include the informational power. It is information intelligence that allows one to govern the societal informational propulsion for the benefit of the progression of Society.

Finally, this information intelligence is particularly essential to the works of codesign around the Information World, the only place that allows one to engage real responsibilities in the informational progression of Society. 
